\title{
Anaesthesia for minimally invasive surgery
}

\author{
Marta Dec, Pawel Andruszkiewicz \\ $2^{\text {nd }}$ Department of Anaesthesiology and Intensive Care, Central Teaching Hospital, Warsaw Medical University, Warsaw, Poland \\ Videosurgery Miniinv 2015; 10 (4): 509-514
}

DOI: 10.5114/wiitm.2015.56411

\begin{abstract}
Minimally invasive surgery (MIS) is rising in popularity. It offers well-known benefits to the patient. However, restricted access to the surgical site and gas insufflation into the body cavities may result in severe complications. From the anaesthetic point of view MIS poses unique challenges associated with creation of pneumoperitoneum, carbon dioxide absorption, specific positioning and monitoring a patient to whom the anaesthetist has often restricted access, in a poorly lit environment. Moreover, with refinement of surgical procedures and growing experience the anaesthetist is presented with patients from high-risk groups (obese, elderly, with advanced cardiac and respiratory disease) who once were deemed unsuitable for the laparoscopic technique. Anaesthetic management is aimed at getting the patient safely through the procedure, minimizing the specific risks arising from laparoscopy and the patient's coexisting medical problems, ensuring quick recovery and a relatively pain-free postoperative course with early return to normal function.
\end{abstract}

Key words: minimally invasive surgery, general anaesthesia, perioperative management.

\section{Introduction}

Minimally invasive surgery (MIS) is rising in popularity. It offers the benefits of less postoperative pain, early ambulation and shorter hospital stay [1] as well as better cosmetic results [2]. On the other hand, restricted access to the surgical site and gas insufflation into the peritoneum/extraperitoneal space may result in severe complications such as pulmonary embolism, pneumothorax, pneumomediastinum, hemodynamic instability and haemorrhage [3].

Since the first cholecystectomy performed by Philippe Mouret in 1987 in Lyons, the list of procedures performed endoscopically has been continuously expanding (Table I) [4].

It must be emphasized, however, that minimally invasive surgery does not mean trivial or easy. From the anaesthetic point of view, it poses unique challenges associated with creation of pneumoperitoneum, carbon dioxide $\left(\mathrm{CO}_{2}\right)$ absorption, specific positioning and monitoring a patient to whom the anaesthetist has often restricted access, in a poorly lit environment. Moreover, with refinement of surgical procedures and growing experience the anaesthetist is presented with patients from high-risk groups (obese, elderly, with advanced cardiac and respiratory disease) who once were deemed unsuitable for laparoscopic technique. Having said that, the anaesthetic management is aimed at getting the patient safely through the procedure, minimizing the specific risks arising from laparoscopy and the patient's coexisting medical problems, ensuring quick recovery and a relatively pain-free postoperative course with early return to normal function.

\section{Preoperative anaesthetic assessment of patients for minimally invasive surgery procedures}

\section{Contraindications}

All patients presenting for minimally invasive surgery must be thoroughly assessed with respect

\section{Address for correspondence}

Pawel Andruszkiewicz MD, PhD, $2^{\text {nd }}$ Department of Anaesthesiology and Intensive Care, Central Teaching Hospital, Warsaw Medical University, 1 A Banacha St, 00-097 Warsaw, Poland, phone: +48 602100 798, e-mail: paul.andruszkiewicz@gmail.com 
Table I. Selected procedures performed laparoscopically

\begin{tabular}{|l|}
\hline Cholecystectomy \\
\hline Adrenalectomy \\
\hline Appendectomy \\
\hline Hernia repair \\
\hline Donor nephrectomy \\
\hline Sleeve gastrectomy \\
\hline Splenectomy \\
\hline Liver resection \\
\hline Colorectal surgery \\
\hline
\end{tabular}

to issues inherent to laparoscopy and the patient's medical status.

The list of contraindications has changed over time as a result of growing experience of surgeons and refinement of the laparoscopic technique.

Preoperative assessment and selection of additional tests is more determined by patients' functional status and comorbidities than the operative technique. On the other hand, the anaesthetist must be aware of the fact that increased intra-abdominal pressure and specific intraoperative positioning may have an impact on both cardiac and respiratory function. According to the 2014 ACC/AHA Guidelines on perioperative cardiovascular evaluation and management of patients undergoing non-cardiac surgery, perioperative tests and treatments should be targeted at specific patient subsets. Routine perioperative stress testing in patients at low risk for cardiac events undergoing low risk elective non-cardiac surgery has no benefit, but it could be harmful by exposing the patient to unnecessary treatments, such as revascularization procedures [5]. A routine preoperative echocardiogram for asymptomatic patients is not indicated.

Patients with right-to left cardiac shunt are at risk of paradoxical embolism and are deemed ineligible for laparoscopic surgery. The situation is less clear in patients with patent foramen ovale since many of them undergo uncomplicated laparoscopic surgery without even realizing they have intracardiac pathology [6].

In patients with advanced pulmonary disease and marginal respiratory reserve, preoperative spirometry and baseline arterial blood gas analysis may be of benefit [7].
The intraoperative course may be more turbulent, because the pre-existing respiratory function impairment is exacerbated by pneumoperitoneum and the steep head-up position, but in comparison to open techniques there are fewer incidents of respiratory dysfunction in the perioperative period [8].

Obesity was once thought to be a contraindication, but it has transformed into a strong indication. In this group in particular quick recovery and early mobilization make the choice of laparoscopy over open technique justified [9].

Caution is advised in patients with increased intracranial pressure particularly if the surgery is planned in a steep head-down position [10].

\section{Effects of pneumoperitoneum and patient position on key vital functions}

\section{Cardiovascular function}

To optimize surgical access, patients are placed in either a head-down (Trendelenburg) or a reverse Trendelenburg position. Several factors may modify the patient's response to intraoperative positioning. Patient's age, coexisting cardiorespiratory disease, volaemic status, anaesthetic agents and degree of table tilt are among those most commonly pointed out in various studies.

Increased intra-abdominal pressure and neuroendocrine vasoactive compounds (catecholamines, vasopressin) released in response to gas insufflation cause an increase in systemic vascular resistance (SVR) and a decrease in preload caused by vena cava inferior compression. Reduction in cardiac filling pressures results in decreased stroke volume (SV) which may lead to a decrease in mean arterial pressure (MAP) [11]. These changes are enhanced in the reverse Trendelenburg position. Hypotension with dangerous cerebral and myocardial hypoperfusion may ensue. This can be mitigated by using the lowest possible insufflation pressures and fluid loading prior to induction of anaesthesia.

The effects of pneumoperitoneum are less pronounced in the Trendelenburg position. Gravity, by enhancing preload, opposes the increase in intra-abdominal pressure maintaining cardiac output.

The reverse Trendelenburg position with pneumoperitoneum increases the risk of deep vein thrombosis due to venous compression and blood pooling in the lower extremities. This mandates 
thrombosis prophylaxis and early postoperative mobilization [12].

Pneumoperitoneum may also have an impact on splanchnic perfusion and organ blood flow. Increased intra-abdominal pressure triggers a reduction in hepatic and renal blood flow, which in turn changes the pharmacokinetics of drugs metabolized by these organs.

\section{Respiratory function}

The pulmonary implications of increased intrathoracic pressure caused by pneumoperitoneum include a decrease in pulmonary compliance and functional residual capacity (FRC) with potential for atelectasis and ventilation/perfusion (V/Q) mismatch. These changes are more pronounced in the head-down position, which results in cephalad diaphragm displacement. Subsequent atelectasis and ventilation/ perfusion mismatch may induce hypoxaemia, which may be mitigated by application of PEEP (positive end-expiratory pressure). The decrease in thoracopulmonary compliance necessitates higher airway pressures to reach targeted tidal volume. Systemic carbon dioxide absorption must be considered in anaesthetic management. Mechanical ventilation should be adjusted accordingly to $\mathrm{CO}_{2}$ absorption to avoid hypercarbia, which may result in hypertension and dysrhythmias.

Since the degree of postoperative respiratory function impairment depends on the size and location of the surgical incision, the compromise is less pronounced after laparoscopy in comparison to laparotomy [13].

\section{Other complications related to patients' positioning for minimally invasive surgery procedures}

The prolonged head-down position may lead to cerebral oedema and elevation in intraocular pressure with potential for perioperative visual loss [14].

Severe airway oedema with post-extubation obstruction was reported after procedures in a steep head-down position [15].

Severe desaturation due to endotracheal tube migration into the main bronchus was also reported [16].

\section{Anaesthetic management}

\section{Preparation}

Good communication among the operating theatre team is an essential condition optimizing coop- eration during the intraoperative management. All relevant details concerning type of the surgery, positioning of the patient and location of the surgical team and equipment must be discussed within the operation team.

In order to avoid postoperative neuropathies particular attention must be paid during the patient's positioning and fastening on the operation table. All potential pressure points should be carefully padded.

Eyes must be covered and protected against abrasions and compression injury. The endotracheal tube needs to be properly secured and its position checked regularly.

\section{Monitoring}

The basic vital functions monitoring encompasses: three lead ECG, non-invasive blood pressure (NIBP), pulse oximetry, capnography, monitoring of neuromuscular block and body temperature. The patient's poor cardiac functional status may warrant extensive haemodynamic monitoring (invasive arterial blood pressure, pulse continuous cardiac output (PICCO) and transoesophageal echocardiography).

Instant access to a serial blood gas monitoring analyzing system may be particularly valuable in differential diagnosis in rapid deterioration of cardiopulmonary function.

Urinary catheterization should be considered for lower abdominal procedures.

\section{Intraoperative management}

Since quick recovery and minimal disruption to everyday routine are desirable targets of MIS, this implies specified requirements from anaesthetic technique as well. Controllable intravenous and volatile anaesthetics with a favourable pharmacokinetic and pharmacodynamic profile are usually used for both induction and maintenance of anaesthesia. Hence the ideal anaesthetic for MIS should have quick onset, short duration of action and should be free of adverse effects. Propofol with its short, context-sensitive half life and new volatile agents (sevoflurane, desflurane) that are poorly soluble in the blood (low blood/gas coefficient) fit these criteria almost perfectly.

Adequate analgesia must be provided to block painful stimuli from the operative field. Controllable opioids are usually preferred due to the requirements of MIS procedures. 
The opioid of choice is remifentanil due to its unique pharmacokinetic properties. Metabolism of remifentanil is independent of the liver and kidney function with a short, context-sensitive half-life, and it does not accumulate within the body even during prolonged procedures.

Fentanyl and sufentanil are viable alternatives.

The unique pharmacokinetic properties of propofol and remifentanil coupled with their ease of titration have made total intravenous anaesthesia (TIVA) the preferred method for minimally invasive surgery in many centres [17]. The antiemetic properties of propofol are particularly noteworthy in the context of increased risk of emesis in laparoscopic procedures. There is no clear advantage of TIVA versus volatile anaesthesia with a short-acting opioid in terms of awakening time [18].

There has been an increased interest in anaesthetic adjuvants such as intravenous lignocaine and $\alpha_{2}$-agonists (dexmedetomidine) as part of the opioid-sparing strategy and a method to attenuate the hemodynamic stress response $[19,20]$. Some authors advocate using regional anaesthesia such as transverse abdominis block (TAP) to decrease the requirement for opioids and minimize their side effects [21].

Good muscle paralysis is mandatory to keep the abdominal cavity compliant to enable gas insufflation and to provide the surgeon with optimal operation conditions [22]. The challenge for the anaesthetist is to keep the patient adequately relaxed almost till the end of the procedure and to allow efficacious spontaneous breathing after the last sutures are placed. This can be achieved by using short-acting non-depolarizing muscle relaxants. The use of neuromuscular monitoring is mandatory in this context. The introduction of new reversal agents such as sugammadex enables termination of deep neuromuscular blockade almost instantly after the end of the procedure [23]. Avoidance of neostigmine - a cholinesterase inhibitor used traditionally to reverse the neuromuscular blockade - can be particularly beneficial due to its strong emetic potential.

To ensure the patient's safety, as well as to provide the operator with optimal surgical conditions, general anaesthesia with endotracheal intubation and controlled ventilation is employed in the majority of cases. The endotracheal tube provides ultimate airway protection and enables efficient ventilation, when higher airway pressures are required to provide desired settings. The use of a laryngeal mask airway (LMA) remains controversial. Second generation supraglottic airway devices (SAD 2) are preferred over the classic LMA since they offer better protection from aspiration and enable higher ventilation pressures [24].

Controlled ventilation is recommended in MIS to provide ventilation suitable to compensate for changes resulting from fluctuations in chest compliance and $\mathrm{CO}_{2}$ absorption. Pressure controlled ventilation modes enable implementation of lower peak pressures to achieve desirable ventilation, as well as better oxygenation due to alveolar recruitment. The beneficial effect of PEEP application to prevent atelectasis should be balanced against its potential impairment of cardiac output.

\section{Postoperative management}

Careful monitoring of the patient's vital functions must be continued in the recovery ward. Sufficient oxygenation is usually provided with the nasal catheter until the patient is discharged to the ward.

Satisfactory postoperative analgesia is of paramount importance. Pain intensity must be regularly evaluated with an objective scale. The visual analogue scale (VAS) and verbal pain score are routinely used for this purpose. A multimodal pain relief approach is usually used, with a combination of paracetamol, non-steroidal anti-inflammatory drugs (NSAIDs) [25], opioids and regional anaesthesia techniques. The latter is achieved by wound and peritoneum infiltration with local anaesthetics [26]. Anticonvulsants such as gabapentin and pregabalin administered perioperatively reduce postoperative pain and opioid consumption without significant side effects $[27,28]$. The multimodal approach provides analgesia by blocking various pain transmitting pathways and enables reduction of doses of individual drugs. Such treatment extends the safety margin and reduces side effects and the complication ratio [29].

Postoperative nausea and vomiting (PONV) can prevent early oral intake and ambulation. The risk factors for PONV occurrence include female gender, previous postoperative nausea and vomiting, non-smoking status, motion sickness and use of opioids, volatiles and cholinesterase inhibitors. The strategy to reduce this complication comprises me- 
ticulous anaesthetic technique aimed at avoiding stomach insufflation, a drug regimen relatively free of emetogenic drugs, and pharmacologic prophylaxis with dexamethasone and ondansetron [30].

\section{Summary}

Laparoscopic techniques have revolutionized surgery. It is hard to think of a procedure that has not at least been attempted in a minimally invasive way. Anaesthetic management must be focused on safe, controllable anaesthesia and provision of quick, pain-free recovery. Uneventful surgery and properly conducted anaesthesia enable early hospital discharge of the patient and return to full functional status. To achieve this goal, good communication and cooperation among all members of the operation team are absolutely essential.

\section{Conflict of interest}

The authors declare no conflict of interest.

\section{References}

1. Grace PA, Quereshi A. Coleman J, et al. Reduced postoperative hospitalization after laparoscopic cholecystectomy. Br J Surg 1991; 78: 160-2.

2. Wroblewski TM, Kobryn K, Nazarewski L, et al. Minilaparoscopic cholecystectomy - the new non-visible scars technique. Preliminary report of first series. Videosurgery Miniinv 2015; 10: 150-4.

3. Chui PT, Gin T, Oh TE. Aanesthesia for laparoscopic general surgery. Anaesth Intensive Care 1993; 21: 163-71.

4. Crozier TA. Introduction. Anaesthesia for minimally invasive surgery. Cambridge University Press, UK 2010; 1-6.

5. Fleisher LA, Fleischmann KE, Auerbach AD, et al. 2014 ACC/ AHA Guidelines on Perioperative Cardiovascular Evaluation and Management of Patients Undergoing Noncardiac Surgery. A report of the American College of Cardiology/ American Heart Association Task Force on practice guidelines. J Am Coll Cardiol 2014; 64: e77-137.

6. Uchida S, Yamamoto M, Masaoka Y, et al. A case of acute pulmonary embolism and acute myocardial infarction with suspected paradoxical embolism after laparoscopic surgery. Heart Vessels 1999; 14: 197-200.

7. van Huisstede A, Biter LU, Luitwieler R, et al. Pulmonary function testing and complications of laparoscopic bariatric surgery. Obes Surg 2013; 23: 1596-603.

8. Nguyen NT, Lee SL, Goldman C, et al. Comparison of pulmonary function and postoperative pain after laparoscopic versus open gastric bypass: a randomized trial. J Am Coll Surg 2001; 192: 469-76.

9. Zollinger A, Krayer S, Singer T, et al. Haemodynamic effects of pneumoperitoneum in elderly patients with an increased cardiac risk. Eur J Anaesth 1997; 14: 266-75.
10. Mobbs RJ, Yang MO. The dangers of diagnostic laparoscopy in the head injured patient. J Clin Neurosci 2001; 9: 592-3.

11. Marshall RL, Jebson PJR, Davie IT, et al. Circulatory effects of carbon dioxide insufflations of the peritoneal cavity for laparoscopy. Br J Anaesth 1972; 44: 680-4.

12. Beebe DS, McNevin MP, Crain JM, et al. Evidence of venous stasis after abdominal insufflations for laparoscopic cholecystectomy. Surg Gynecol Obstet 1993; 176: 443-7.

13. Scauer PR, Luna J, Ghiatas AA, et al. Pulmonary function after laparoscopic cholecystectomy. Surgery 1993; 114: 389-97.

14. Pinkney TD, King AJ, Walter C. Raised intraocular pressure (IOP) and perioperative visual loss in laparoscopic colorectal surgery: a catastrophe waiting to happen? A systematic review of evidence from other surgical specialities. Tech Coloproctol 2012; 16: 331-5.

15. Hayden P, Cowman S. Anaesthesia for laparoscopic surgery. CEACCP 2011; 11: 177-80

16. Labato EB, Paige GB, Brown MM, et al. Pneumoperitoneum as a risk factor for endobronchial intubation during laproscopic gynecologic surgery. Anesth Analg 1998; 86: 301-3.

17. Crozier TA. Anaesthesia for laparoscopic surgery. Anaesthesia for minimally invasive surgery. Cambridge University Press UK 2010; 56-74.

18. Grundmann U, Silomon M, Bach F, et al. Recovery profile and side effects of remifentanyl-based anaesthesia with desflurane or propofol for laparoscopic cholecystectomy. Acta Anaesthesiol Scand 2001; 45: 320-6.

19. Jain S, Khan RM. Effect of peri-operative intravenous infusion of lignocaine on haemodynamic responses to intubation, extubation and post-operative analgesia. Indian J Anaesth 2015; 59: 342-7.

20. Anjum N, Tabish H, Debdas S, et al. Effects of dexmedetomidine and clonidine as propofol adjuvants on intra-operative hemodynamics and recovery profiles in patients undergoing laparoscopic cholecystectomy: a prospective randomized comparative study. Avicenna J Med 2015; 5: 67-73.

21. Conaghan P, Maxwell-Armstrong C, Bedforth N, et al. Efficacy of transversus abdominis plane blocks in laparoscopic colorectal resections. Surg Endosc 2010; 24: 2480-4.

22. Van Wijk RM, Watts RW, Ledowski T, et al. Deep neuromuscular block reduces intra-abdominal pressure requirements during laparoscopic cholecystectomy: a prospective observational study. Acta Anaesthesiol Scand 2015; 59: 434-40.

23. Fields AM, Vadivelu N. Sugammadex: a novel neuromuscular blocker binding agent. Curr Opin Anaesthesiol 2007; 20: 307-10.

24. Lim Y, Goel S. Proseal is effective alternative to laryngoscope guided tracheal intubation. Anaesth Intensive Care 2007; 35: 52-6.

25. Boccara G, Chaumeron A, Pouzeratte Y, Mann C. The preoperative administration of ketoprofen improves analgesia after laparoscopic cholecystectomy in comparison with propacetamol or postoperative ketoprofen. $\mathrm{Br} J$ Anaesth 2005; 94: 347-51.

26. Cha SM, Kang H, Baek CW, et al. Peritrocal and intraperitoneal ropivacaine for laparoscopic cholecystectomy: a prospective, randomized, double-blind controlled trial. J Surg Res 2012; 175: 251-8.

27. Hassani V, Pazouki A, Nikoubakht N, et al. The effect of gabapentin on reducing pain after laparoscopic gastric bypass 
surgery in patients with morbid obesity: a randomized clinical trial. Anesth Pain Med 2015; 5: e22372.

28. Bekawi MS, El Wakeel LM, Al Taher WM, et al. Clinical study evaluating pregabalin efficacy and tolerability for pain management in patients undergoing laparoscopic cholecystectomy. Clin J Pain 2014; 30: 944-52.

29. Elvir-Lazo OL, White PF. The role of multimodal analgesia in pain management in ambulatory surgery. Curr Opin Anaesthesiol 2010; 23: 697-703.

30. Wong JJ, Ho ST, Liu YH, et al. Dexamethasone reduces nausea and vomiting after laparoscopic cholecystectomy. Br J Anaesth 1999; 83: 772-5.

Received: 25.11.2015, accepted: 5.12.2015. 